\title{
Solid-Boundary Treatment for Moment Equations
}

\author{
Loc Khieu* and Bram van Leer ${ }^{\dagger}$ \\ Department of Aerospace Engineering \\ The University of Michigan, Ann Arbor, MI 48109-2140, USA
}

\begin{abstract}
We present progress in formulating accurate solid-wall boundary conditions for hyperbolic-relaxation systems of moment equations. This process uses detailed information about the molecular velocity distribution in the flow domain next to the boundary. Results for Couette flow are validated by BGK solutions of the Boltzmann equation.
\end{abstract}

\section{Introduction}

The research reported here is part of our ambitious project "CFD by First-Order PDEs." We aimed at modeling all flows, except free-molecular flow, by systems of hyperbolicrelaxation equations, obtained by taking moments of the Boltzmann equation following the procedure suggested by Grad! 1 As a result, a single code based on this system could simulate flows up to intermediate Knudsen numbers, and would be preferable to a Navier-Stokes code if hybridization with a DSMC code were needed.

Comparing to the Navier-Stokes system, this approach has many potentially physical and numerical advantages. Regarding numerical issues: accuracy problems on adaptive unstructured grids can be avoided because there are no second or higher derivatives to be approximated. The source terms, despite their stiffness, are only local. In addition, the compact stencils facilitate code parallelization. Regarding physical issues: more flow phenomena could be captured due to the ability to have a larger set of independent flow

${ }^{*}$ Graduate Student Research Assistant, Student Member AIAA. Email: khieuhl@umich.edu

${ }^{\dagger}$ Professor, Fellow AIAA.

Copyright (c) 2011 by Loc Khieu and Bram van Leer. Published by the American Institute of Aeronautics and Astronautics, Inc. with permission. 
variables in the system of governing equations. A typical example is that stresses and heat fluxes are treated as independent quantities; in the Navier-Stokes system, these are related back to velocity gradients and the temperature gradient by approximate-in-nature constitutive equations.

As much as we wish this were not the case, the moment approach is not yet fully mature. It was shown in $2 \sqrt[3]{3}$ that the approach works remarkably well for subsonic and transonic flows. For higher-speed flows, however, it is unable to obtain an accurate representation of shock structure, thus rendering it unsuited for supersonic and hypersonic applications.

At the moment, efforts are concentrated on making moment systems work up to their full potential for low-speed rarefied gas applications, in particular flows through and around MEMS devices. In one research direction, highly effective numerical schemes for integrating a hyperbolic-relaxation system were developed. The findings were reported in our previous paper, ${ }^{[4}$ and more details in Suzuki's Ph.D. thesis. ${ }^{[3}$ In another research direction, detailed in Section [I], we are working on an accurate formulation of solid-wall boundary conditions for the moment system. The boundary treatment uses detailed information about the molecular velocity distribution of the gas near the wall, as well as the boundary's parameters, i. e. temperature $T^{\mathrm{w}}$, velocity $\mathbf{u}^{\mathrm{w}}$, and surface roughness.

Section III describes our numerical computation of BGK solutions of the Boltzmann equation. Comparison between these solutions and ones obtained from Grad's 13-moment system $^{1}$ for linearized Couette flow will be presented in Section IV. Section $V$ concludes with observations and remarks.

\section{Solid-boundary treatments}

In this section we first discuss Grad's approach ${ }^{1}$ to formulating boundary conditions for a gas described by a system of moments of Boltzmann's equation. We demonstrate an inconsistency in his boundary treatment, and propose two alternative formulations.

\section{II.A. Grad's formulation and its inconsistency}

For a system of moment equations in extended hydrodynamics, Grad [1, p. 379] proposed a now classical method to formulate solid-boundary conditions based on Maxwell's kinetic 
boundary condition. In this approach the velocity-distribution function or, for short, distribution function, $f\left(v_{x}, v_{y}, v_{z}\right)$ of molecules in the Knudsen layer is a linear combination of the distribution functions of the incident molecules, $f^{-}\left(v_{x}, v_{y}, v_{z}\right)$, and the reflected molecules, $f^{+}\left(v_{x}, v_{y}, v_{z}\right)$. The total velocity vector of a molecule $\mathbf{v}=\left\{v_{x}, v_{y}, v_{z}\right\}$ is the sum of the molecule's thermal velocity $\mathbf{c}$ and the average fluid velocity $\mathbf{u}$, that is, $\mathbf{v}=\mathbf{c}+\mathbf{u}$. The superscript "-" indicates that molecules in this class travel in the direction opposite to the boundary normal, making their normal velocity component negative; similarly, the superscript "+" indicates molecules moving in the direction of the normal, thus having positive normal velocity. In what follows the normal direction will be denoted by subscript " $y$." By definition, $f^{-}\left(v_{x}, v_{y}, v_{z}\right)=0$ for $v_{y}>0$ and $f^{+}\left(v_{x}, v_{y}, v_{z}\right)=0$ for $v_{y}<0$.

For any moment model, the velocity distribution of molecules in the flow domain far away from the boundary is in a known form, hence the form of $f^{-}$is also known. The distribution function $f^{+}$is more complicated because molecules in this class experience collisions with the boundary. One possibility is that the molecule is reflected specularly after collision; this type of collision reverses the normal component of the molecule's momentum, everything else remains unchanged. Thus, the distribution function for this class is the mirror image of the one before collision, $f^{-}\left(v_{x}, v_{y}, v_{z}\right)$ with respect to the plane $\left(v_{y}=0\right)$; hence $f_{\text {specular }}^{+}=f^{-}\left(v_{x},-v_{y}, v_{z}\right)$. Due to roughness of the boundary surface, there is a possibility that molecules experiences enough collisions at the boundary to reach equilibrium before being reflected back into the flow; they may then be assumed to have acquired a Maxwellian distribution function, $f_{\text {diffusive }}^{+}=f_{\mathrm{M}}^{\mathrm{w}}\left(v_{x}, v_{y}, v_{z}\right)$. This type of reflection is called diffusive reflection.

The overall distribution function at the wall is expressed as:

$$
\begin{aligned}
f\left(v_{x}, v_{y}, v_{z}\right) & =f^{-}+f^{+}=f^{-}+\left[\sigma C f_{\text {diffusive }}^{+}+(1-\sigma) f_{\text {specular }}^{+}\right] \\
=f^{-}\left(v_{x}, v_{y}, v_{z}\right) & +\left[\sigma C f_{\mathrm{M}}^{\mathrm{w}}\left(v_{x}, v_{y}, v_{z}\right)+(1-\sigma) f^{-}\left(v_{x},-v_{y}, v_{z}\right)\right] .
\end{aligned}
$$

Here $\sigma \in[0,1]$, the accommodation factor, expresses how likely a molecule will be diffusively reflected after collision with the boundary; $f_{\mathrm{M}}^{\mathrm{w}}\left(v_{x}, v_{y}, v_{z}\right)$ carries information about the temperature $T^{\mathrm{w}}$ and velocity $\mathbf{u}^{\mathrm{w}}$ of the wall in the following form

$$
f_{\mathrm{M}}^{\mathrm{w}}\left(v_{x}, v_{y}, v_{z}\right)=\frac{\rho}{\left(2 \pi R T^{\mathrm{w}}\right)^{3 / 2}} \exp \left[-\frac{\left(\mathbf{v}-\mathbf{u}^{\mathrm{w}}\right) \cdot\left(\mathbf{v}-\mathbf{u}^{\mathrm{w}}\right)}{2 R T^{\mathrm{w}}}\right] .
$$


Without loss of generality it may be assumed that the solid boundary does not move in any direction, i. e. $\mathbf{u}^{\mathrm{w}}=0$. The coefficient $C$ is yet unknown, to be determined by the boundary condition of non-penetration, or zero normal mass-flux:

$$
\iint_{-\infty}^{+\infty} \int_{y} f\left(v_{x}, v_{y}, v_{z}\right) d v_{x} d v_{y} d v_{z} \equiv\left\langle v_{y} f\right\rangle=0 .
$$

The value of $C$ thus calculated completes $f\left(v_{x}, v_{y}, v_{z}\right)$. To calculate any macroscopic or average quantity at the wall for boundary condtions, the corresponding moment of the full distribution function $f\left(v_{x}, v_{y}, v_{z}\right)$ given by (1) must be taken:

$$
\begin{aligned}
\langle w(\mathbf{v}) f\rangle= & \int_{-\infty}^{+\infty}\left[\int_{-\infty}^{0} w(\mathbf{v}) f^{-}\left(v_{x}, v_{y}, v_{z}\right) d v_{x}\right] d v_{y} d v_{z} \\
& +\sigma C \iint_{-\infty}^{+\infty}\left[\int_{0}^{+\infty} w(\mathbf{v}) f_{\mathrm{M}}^{\mathrm{w}}\left(v_{x}, v_{y}, v_{z}\right) d v_{x}\right] d v_{y} d v_{z} \\
& +(1-\sigma) \int_{-\infty}^{+\infty}\left[\int_{0}^{+\infty} w(\mathbf{v}) f^{-}\left(v_{x},-v_{y}, v_{z}\right) d v_{x}\right] d v_{y} d v_{z} .
\end{aligned}
$$

The choice of weight function $w(\mathbf{v})$ determines what macroscopic quantity will be calculated. For example, tangential velocity components are calculated by using $w(\mathbf{v})=v_{i}, i=\{x, z\}$; the pressure tensor $P_{i j}$ is calculated from moment with weight function $w(\mathbf{v})=v_{i} v_{j},(i, j)=$ $\{x, y, z\}$; etc.

Unfortunately, the set of boundary conditions obtained from this procedure is inconsistent. For most flow variables of interest there is more than one expression to describe their behavior at the boundary, and there are not enough constraints to identify the correct one. In our previous papers, $\frac{4,5}{5}$ these inconsistencies were demonstrated using two moment models: the Gaussian 10-moment model and Grad's 10-moment model. It must be mentioned that these two 10-moment models may not accurately capture the flow physics near the wall. Those models are among the simplest of moment approaches, thus easier to study analytically; yet they are mathematically fairly similar to more realistic models as the same principle is used to formulate them. Therefore, it was preferable to use those two models for illustrative purposes. 


\section{II.B. Alternative approaches to formulating solid-boundary conditions}

The inconsistency described in II.A is caused by a discrepancy between the number of constraints, implicit and explicit, acting on (1) and the number of useful degrees-of-freedom it possesses. Local average quantities in the Knudsen layer are utilized in the distribution function $f^{-}$of incident molecules. This makes that local average quantities will appear on the right-hand side of (4), while its left-hand side, by definition, is also one local average quantity. When (4) is used to compute average quantities at the wall for boundary conditions, the expressions obtained are interconnected because one local quantity can appear in multiple expressions. This is technically equivalent to imposing additional conditions on the distribution function (1). However, there is no available freedom to satisfy these new conditions; the only one degree-of-freedom in Grad's formulation has already been used to satisfy the non-penetration condition.

Next are our attempts to circumvent this inconsistency.

\section{II.B.1. 1-C solid-boundary condition model}

Technically, there are two ways to remedy this inconsistency problem. Either we add more degrees of freedom, or dofs, or eliminate all those extra implicit constraints. The former option is a complex procedure. The first step is to find out how many more constraints are imposed implicitly. This depends strongly on which moment system is studied. It is known that, for fluid dynamics, a useful moment system has at least ten variables. We might have to add that many dofs to (1) to satisfy additional constraints. Two dofs can be added before $f^{-}$and $f_{\text {specular }}^{+}$in (1). Others would have to be included inside of the expressions for the components of the distribution function. To derive those expressions from physical principle is complicated; clearly, adding additional dofs is not to be taken lightly.

The second option, in contrast to the first one, produces a common procedure for all moment systems. To eliminate all additional constraints, distribution functions of incident molecules and specularly reflected molecules should be constituted from known average quantities, denoted by superscript “ ," instead of unknown local ones as in the original approach. The former is a physically plausible assumption. The Knudsen layer has a thickness only in the order of a mean-free-path. Molecules are likely to travel collisionless within this 
layer; the dominant collisions are molecule-surface interactions. Thus, there is good chance that incident molecules do not adapt instantly to local condition; their prior properties are unchanged until they collide with the solid-boundary.

All subsequent steps are similar to those in Grad's original formulation. The notable change is that eqn. (4) becomes a recipe for computing flow quantities at the solid boundary in terms of known quantities of incident molecules, and parameters of the solid boundary. The previous couplings are removed. This formulation is a direct implementation of Maxwell's kinetic solid-boundary condition for the Boltzmann equation. We call this the "1Coefficient", or " $1-C$ " boundary model from now on for a reason that will become obvious later.

\section{II.B.2. 2-C solid-boundary condition model}

The second alternative treatment is an extension of the $1-C$ model. We suggest to impose a new condition on $f\left(v_{x}, v_{y}, v_{z}\right)$ besides non-penetration; it is a normalization condition:

$$
\langle f / \rho\rangle \equiv\langle\widehat{f}\rangle=1
$$

This requires further explanation. The distribution function $f\left(v_{x}, v_{y}, v_{z}\right) d \mathbf{v}$ expresses the mass density of molecules having a total velocity in the range of $\mathbf{v} \rightarrow \mathbf{v}+d \mathbf{v}$, at an arbitrary space-time location. On the other hand, the distribution function $\widehat{f}=f / \rho$ carries no information about mass density; $\widehat{f}\left(v_{x}, v_{y}, v_{z}\right) d \mathbf{v}$ expresses the probability to find a molecule having its velocity in the range $\mathbf{v} \rightarrow \mathbf{v}+d \mathbf{v}$, at an arbitrary space-time location. All molecules in the flow field have a real velocity vector; thus there is $100 \%$ certainty to find a molecule with its velocity in the range of $\mathbf{v}$ from $-\infty$ to $+\infty$. This leads to making the normalization condition (5) a fundamental requirement.

To satisfy the normalization condition, an additional $d o f$ in the form of a coefficient $C_{1}$ is introduced into the representation for incident molecules; the coefficient $C$ in diffusive reflection term is renamed $C_{2}$. This creates the $2-C$ model; the one with one dof is called the 1-C model. The coefficient $C_{1}$ must also appear in the expression for specular reflection as this type of reflection does not change anything but the sign of the normal component of momentum. The overall expression for the distribution function change from (1) to (6); 
other details are as in the $1-C$ model.

$$
f\left(v_{x}, v_{y}, v_{z}\right)=C_{1} f^{-}\left(v_{x}, v_{y}, v_{z}\right)+\left[\sigma C_{2} f_{\mathrm{M}}^{\mathrm{w}}\left(v_{x}, v_{y}, v_{z}\right)+(1-\sigma) C_{1} f^{-}\left(v_{x},-v_{y}, v_{z}\right)\right] .
$$

\section{Solving the Boltzmann-BGK equation}

A system of moment equations is derived by taking a finite number of moments of the Boltzmann-BGK equation, or Krook equation. The amount of information contained in the resulting system is just a small subset of what the Boltzmann-BGK equation can describe. Thus, to validate results from the moment approach, solutions of the original equation are the best candidates.

The Boltzmann-BGK equation has the following form in 2-D geometry,

$$
\frac{\partial f}{\partial t}+v_{x} \frac{\partial f}{\partial x}+v_{y} \frac{\partial f}{\partial y}=-\frac{f-f_{\mathrm{M}}}{\tau},
$$

where $f$ is a function of six variables $\left(t, x, y, v_{x}, v_{y}, v_{z}\right)$, and the relaxation time $\tau$ is calculated from other flow quantities as $\tau=\mu / p$. For a 2-D flow geometry, we are not interested in the $z$-direction, as every flow quantity is assumed uniform in that direction. However, when one solves (7), the $v_{z}$-dimension still has to be discretized into $N_{v_{z}}$ small intervals; the phase-space mesh is $N_{v_{z}}$ times larger than it needs to be, and makes an already-expensive calculation even more prohibitive.

To make the calculation more feasible, we utilize the reduction approach proposed by Chu $\sqrt[6]{7}$ Instead of solving a single equation that depends on a 5 -D phase space of $\left(x, y, v_{x}, v_{y}, v_{z}\right)$, we solve a system of two equations having a similar mathematical form but depending only on a 4 -D phase space $\left(x, y, v_{x}, v_{y}\right)$. This substitution easily reduces the total number of unknowns by an order of magnitude. The system to solve is

$$
\frac{\partial \boldsymbol{\Phi}}{\partial t}+v_{x} \frac{\partial \boldsymbol{\Phi}}{\partial x}+v_{y} \frac{\partial \boldsymbol{\Phi}}{\partial y}=-\frac{\boldsymbol{\Phi}-\boldsymbol{\Phi}_{\mathrm{M}}}{\tau}
$$

where $\mathbf{\Phi}=\left(\phi_{0}, \phi_{1}\right)$ is a function of $\left(t, x, y, v_{x}, v_{y}\right)$. Its components are related to the original 
distribution function $f$ in (7) by the following integrals:

$$
\begin{aligned}
& \phi_{0}\left(t, x, y, v_{x}, v_{y}\right)=\int_{-\infty}^{+\infty} f\left(t, x, y, v_{x}, v_{y}, v_{z}\right) d v_{z}, \\
& \phi_{1}\left(t, x, y, v_{x}, v_{y}\right)=\frac{1}{2} \int_{-\infty}^{+\infty} v_{z}^{2} f\left(t, x, y, v_{x}, v_{y}, v_{z}\right) d v_{z} .
\end{aligned}
$$

$\phi_{0}$ and $\phi_{1}$ carry information about density and total energy in the $z$ - direction, respectively. Any macroscopic quantity is a linear combination of appropriate moments of $\phi_{0}$ and $\phi_{1}$.

We solve system (8) by a second-order finite-volume scheme with Runge-Kutta 2-stage time marching. The slopes of the solution in both physical and velocity spaces are reconstructed from data in neighboring cells. As there is no advection happening in velocity space, formally we don't have to use a limiter there. In our experience, not using any type of limiter on the distribution function in velocity space will reduce the total number of velocity cells necessary to achieve conservation of energy, which is the most difficult to accomplish among all three conservation laws. No limiter is used in physical space either because we expect smooth solutions for Couette flow.

Concerning solid-boundary conditions for the Boltzmann-BGK equation, we also employ two different models, called " $1-C$ " and " $2-C$ ". They are conceptually similar to those for the moment system, hence we use the same names. The only difference is that the distribution of incident molecules, $f^{-}$, is the solution itself. In a moment system, it is calculated from average quantities.

\section{Simulation of Couette flow}

To test the proposed alternative boundary treatment, a simple quasi-one-dimensional linearized Couette flow is solved for Knudsen number ranges from 0.01 to 10 , covering both continuum-transition regime and transition regime. Geometry and coordinate system for the flow are shown in Figure 1. Solution from Grad's 13-moment will be compared to solutions of the reduced Boltzmann-BGK system, and those of the Navier-Stokes with 1st-order slip solid-boundary condition. The familiar Lees's analytical approximate solution is formally

identical to results of the Navier-Stokes with 1st-order slip [8, p. 431]. Because all three solutions will be mentioned frequently in the next part, they will be called by their shorten

$$
8 \text { of } 15
$$



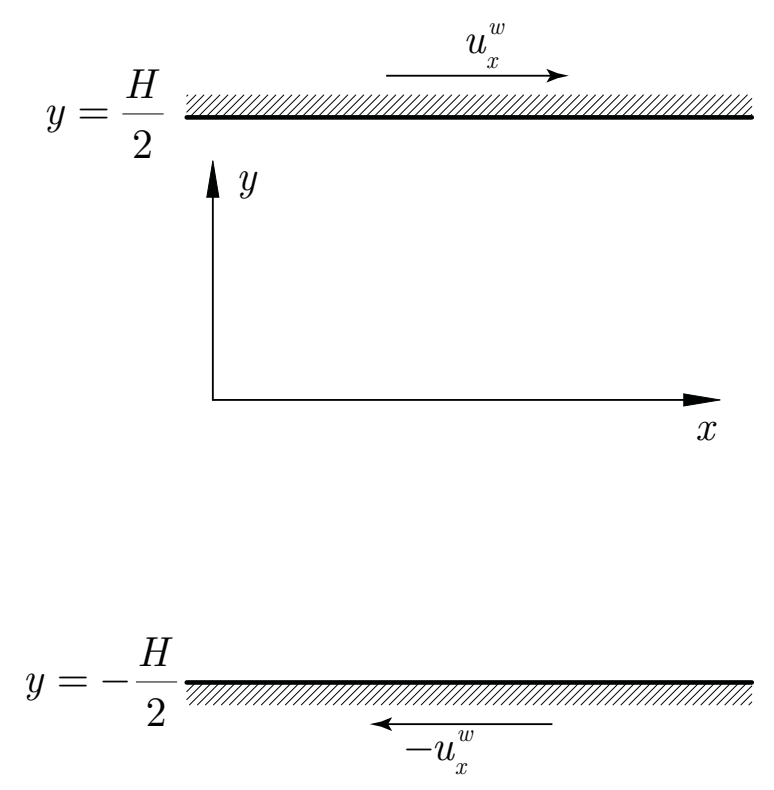

Figure 1: Couette-flow geometry and coordinate system.

names as 13M-sol, BGK-sol, and NS-sol. Among them, BGK-sols are considered the most accurate.

The moment system is only valid for monatomic gas; therefore, we choose argon as the working medium. Gas-surface interaction is fully diffusive, $\sigma=1$. However, all formulae for boundary conditions are derived with an arbitrary value of $\sigma$. The walls move at very low speed, $M_{\mathrm{w}}=0.01$ to satisfy condition 10 for linearizability 8, p. 424]

$$
\frac{\left(u_{y}^{\mathrm{w}}\right)^{2}}{2 R T^{\mathrm{w}}} \ll 1
$$

\section{IV.A. Grad's 13-moment system and its solid-boundary conditions}

In this moment system, the underlying distribution function is a function of thirteen average quantities: density $\rho$, average velocity vector $\mathbf{u}$, pressure tensor $\mathbf{P}$, and heat-flow vector q. The pressure tensor is also expressed as a combination of pressure $p=P_{j j} / 3$ and nonequilibrium quantities $p_{i j}$, which relate to shear stresses $\tau_{i j}$ in the Navier-Stokes description by $p_{i j}=-\tau_{i j}$. In 3 -D geometry, this distribution function has the following form

$$
f_{\mathrm{Grad} 13}\left(v_{x}, v_{y}, v_{z}\right)=\frac{\rho}{(2 \pi R T)^{3 / 2}} \exp \left[-\frac{\mathbf{c} \cdot \mathbf{c}}{2 R T}\right]\left[1+\frac{p_{i j}}{2 p R T}-\frac{q_{i} c_{i}}{p R T}\left(1-\frac{\mathbf{c} \cdot \mathbf{c}}{R T}\right)\right] .
$$


The corresponding governing system is a system of thirteen hyperbolic-relaxation PDEs:

$\frac{\partial}{\partial t}\left[\begin{array}{c}\rho \\ \rho u_{i} \\ \rho u_{i} u_{j}+P_{i j} \\ \rho u_{i} u^{2}+3 u_{i} p+2 u_{j} P_{i j}+2 q_{i}\end{array}\right]$

$+\frac{\partial}{\partial x_{k}}\left[\begin{array}{c}\rho u_{k} \\ \rho u_{i} u_{k}+P_{i k} \\ \rho u_{i} u_{j} u_{k}+\left(u_{i} P_{j k}+u_{j} P_{i k}+u_{k} P_{i j}\right)+S_{i j k} \\ u_{k}\left(\rho u_{i} u^{2}+3 u_{i} p\right)+2 u_{i} u_{j} P_{j k}+2 u_{j} u_{k} P_{i j}+u^{2} P_{i k}+2\left(u_{i} q_{k}+u_{k} q_{i}+u_{j} S_{i j k}\right)+Q_{i k}\end{array}\right]$

$=-\frac{1}{\tau}\left[\begin{array}{c}0 \\ 0 \\ p_{i j} \\ 2 u_{j} p_{i j}+2 q_{i}\end{array}\right]$

where $S_{i j k}=2\left(q_{i} \delta_{j k}+q_{j} \delta_{i k}+q_{k} \delta_{i j}\right), Q_{i j}=R T\left(7 P_{i j}-2 p \delta_{i j}\right)$. For Couette-flow geometry, it reduces to a quasi-one-dimensional system of nine variables: $\rho, u_{x}, u_{y}, P_{x x}, P_{y y}, P_{z z}, P_{x y}$, $q_{x}$, and $q_{y}$.

The system is solved by a second-order finite-volume scheme, without limiter, for spatial discretization. Time integration scheme is the Runge-Kutta 2-stage, similarly to the one employed to solve the reduced Boltzmann-BGK system. The numerical fluxes at cell- interface are calculated by a special flux-vector spitting, which takes advantage of how every term in 12 is a moment of (11) with an appropriate weight. For Couette flow, the numerical fluxes at interface $(j+1 / 2)$ in the $y$-direction are calculated by

$$
\mathbf{G}=\left\langle v_{y} \mathbf{W} f_{j+1 / 2}^{\text {upper }}\right\rangle^{-}+\left\langle v_{y} \mathbf{W} f_{j+1 / 2}^{\text {lower }}\right\rangle^{+}
$$

The weight vector is $\mathbf{W}\left(v_{x}, v_{y}, v_{z}\right)=\left[1, v_{x}, v_{y}, v_{x}^{2}, v_{y}^{2}, v_{z}^{2}, v_{x} v_{y}, v_{x} \mathbf{v}^{2}, v_{y} \mathbf{v}^{2}\right]^{\mathrm{T}}$. The operator \langle\rangle$^{ \pm}$ is the integral over half of three-dimensional velocity space:

$$
\langle f\rangle^{-}=\iint_{+\infty}^{-\infty} \int_{-\infty}^{0} f \mathrm{~d} v_{y} \mathrm{~d} v_{x} \mathrm{~d} v_{z} \quad\langle f\rangle^{+}=\iint_{+\infty}^{-\infty} \int_{0}^{+\infty} f \mathrm{~d} v_{y} \mathrm{~d} v_{x} \mathrm{~d} v_{z} .
$$

10 of 15 
To calculate the numerical fluxes at boundaries, one method is to create ghost cells, assign appropriate values for their variables, then solve the Riemann problem by the described flux-vector splitting. Alternatively, they can be calculated from integrating the distribution function representing the state of the gas at the boundaries with weight $\mathbf{W}$. The latter is selected because the required distribution is readily available, courtesy of the procedures described in II.B. It has the following form, which is (6) rewritten to exclude density information:

$$
\widehat{f}\left(v_{x}, v_{y}, v_{z}\right)=C_{1} \widehat{f}^{-}\left(v_{x}, v_{y}, v_{z}\right)+\left[\sigma C_{2} \widehat{f}_{\mathrm{M}}^{\mathrm{W}}\left(v_{x}, v_{y}, v_{z}\right)+(1-\sigma) C_{1} \widehat{f}^{-}\left(v_{x},-v_{y}, v_{z}\right)\right]
$$

with

$$
\begin{aligned}
& \widehat{f}^{-}\left(v_{x}, v_{y}, v_{z}\right)=\frac{1}{(2 \pi R \widetilde{T})^{3 / 2}} \exp \left[-\frac{(\mathbf{v}-\widetilde{\mathbf{u}})^{2}}{2 R \widetilde{T}}\right]\left[1+\frac{\widetilde{p}_{i j}}{2 \widetilde{p} R \widetilde{T}}-\frac{\widetilde{q}_{i} c_{i}}{\widetilde{p} R \widetilde{T}}\left(1-\frac{(\mathbf{v}-\widetilde{\mathbf{u}})^{2}}{R \widetilde{T}}\right)\right], \\
& \widehat{f}_{\mathrm{M}}^{\mathrm{w}}\left(v_{1}, v_{2}, v_{3}\right)=\frac{1}{\left(2 \pi R T^{\mathrm{w}}\right)^{3 / 2}} \exp \left[-\frac{\left(\mathbf{v}-\mathbf{u}^{\mathrm{w}}\right)^{2}}{2 R T^{\mathrm{w}}}\right] .
\end{aligned}
$$

For the 1-C model, condition (3) is used to calculate the value of $C_{2} ; C_{1}$ is unity. For the 2- $C$ model, both (3) and (5) are used. Results for $C_{1}$ and $C_{2}$ are listed in table 1. In our simulation, values of " $\sim$ " quantities are extrapolated from the interior solution to $y= \pm H / 2$. Then, the numerical fluxes at the boundaries are calculated by:

$$
\begin{aligned}
\mathbf{G}^{\mathbf{w}} & =\iint_{-\infty}^{+\infty} v_{y} \mathbf{W} \widetilde{\rho} \widehat{f} \mathrm{~d} v_{x} \mathrm{~d} v_{y} \mathrm{~d} v_{z} \\
& =C_{1}\left\langle v_{y} \mathbf{W} \widehat{f}^{-}\right\rangle^{-}+\sigma C_{2}\left\langle v_{y} \mathbf{W} \widehat{f}_{\mathrm{M}}^{\mathrm{W}}\right\rangle^{+}+(1-\sigma) C_{1}\left\langle v_{y} \mathbf{W} \widehat{f}^{-}\left(-v_{y}\right)\right\rangle^{+}
\end{aligned}
$$

\section{IV.B. Numerical results}

Figure 2a shows the profiles of velocity for four Knudsen numbers, increasing from right to left. At $K n=0.01$, all three solutions are on top of each other. For the next two values 0.1 and 1, the moment system has a better prediction for the amount of slip at the wall than the Navier-Stokes equations. However, slopes of the velocity profiles predicted by the moment system are worse than NS-sols. For $K n=0.1$, NS-sol has approximately the same velocity gradient as BGK-sol, while $13 \mathrm{M}$-sol is slightly smaller. At $K n=1$, both velocity gradients of 13M-sol and NS-sol are quite far away from BGK-sol. It is interesting that, at 


$$
\begin{aligned}
& C_{1} \quad C_{2} \\
& \text { 1-C model } \quad 1 \quad-\frac{\left\langle v_{y} \widehat{f}^{-}\right\rangle^{-}}{\left\langle v_{y} \widehat{f}_{\mathrm{M}}^{\mathrm{w}}\right\rangle^{+}} \\
& \text {2-C model } \quad-\frac{\left\langle v_{y} \widehat{f}_{\mathrm{M}}^{\mathrm{w}}\right\rangle^{+}}{\Delta} \quad \frac{\left\langle v_{y} \widehat{f}^{-}\right\rangle^{-}}{\Delta} \\
& \Delta=-(2-\sigma)\left\langle\widehat{f}^{-}\right\rangle^{-}\left\langle v_{y} \widehat{f}_{\mathrm{M}}^{\mathrm{w}}\right\rangle^{+}+\sigma\left\langle\widehat{f}_{\mathrm{M}}^{\mathrm{w}}\right\rangle^{+}\left\langle v_{y} \widehat{f}^{-}\right\rangle^{-} \\
& \left\langle\widehat{f}^{-}\right\rangle^{-}=\frac{1}{2}+\sqrt{\frac{R \widetilde{T}}{2 \pi}} \frac{\widetilde{q_{y}}}{5 \widetilde{p} R \widetilde{T}} \quad\left\langle\widehat{f}_{\mathrm{M}}^{\mathrm{w}}\right\rangle^{+}=\frac{1}{2} \\
& \left\langle v_{y} \widehat{f}^{-}\right\rangle^{-}=-\frac{1}{2} \sqrt{\frac{R \widetilde{T}}{2 \pi}}\left(1+\frac{\widetilde{P}_{y y}}{\widetilde{p}}\right) \quad\left\langle v_{y} \widehat{f}_{\mathrm{M}}^{\mathrm{w}}\right\rangle^{+}=\sqrt{\frac{R T^{\mathrm{w}}}{2 \pi}}
\end{aligned}
$$

Table 1: Values of $C_{1}$ and $C_{2}$ for solid-boundary conditions of Grad's 13-moment system

$K n=10$, the moment system predicts a smaller slip than BGK-sol, while it is larger for all other cases. Currently, at this Knudsen number, our calculation for any finer grid runs into a limit cycle, suspending further investigation.

In Figure 2b, $P_{x y}(y=0)$ is plotted against Knudsen numbers. The reason to plot $P_{x y}$ at that location will be explained later. The moment system consistently predicts a smaller shear stress than BGK-sol, while NS-sol is consistently higher. At $K n=10$, the difference between 13M-sol and BGK-sol is quite noticeable, while it is insignificant for smaller Knudsen numbers.

Figure 3 displays the ratio $P_{x y}(y) / P_{x y}(0)$ for all calculations. It shows that profiles of $P_{x y}$ from BGK-sols are uniform for all Knudsen numbers. NS-sols are not shown as their $P_{x y}$ profiles are also uniform, similar to BGK-sols. $P_{x y}$ profiles from $13 \mathrm{M}$-sol are not strictly uniform like BGK-sols or NS-sols; they are uniform only in the middle of the channel. The value of $P_{x y}$ in the boundary cell is always less than the middle cell by a small amount, 


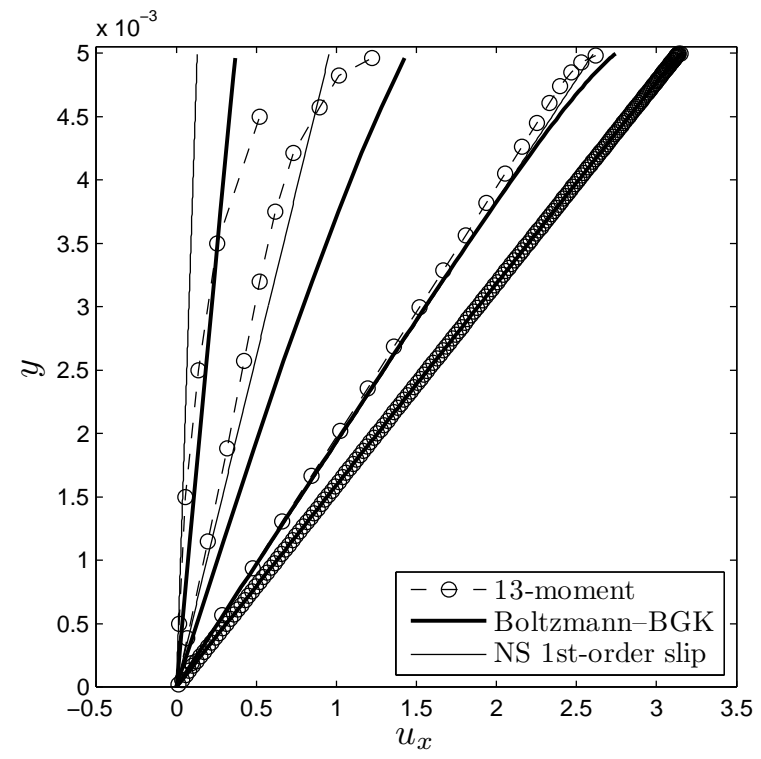

(a) $u_{x}$ profile. $K n=0.01 \rightarrow 10$ from right to left.

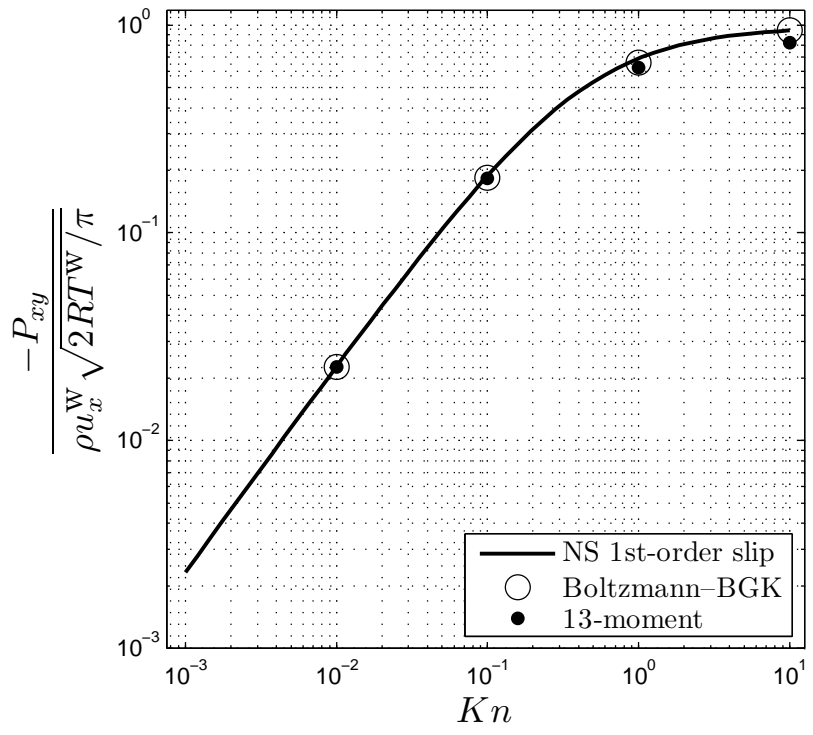

(b) Normalized $P_{x y}$ vs. $K n$

Figure 2: Solutions from the 13-moment system with 1-C boundary condition, the reduced Boltzmann-BGK system, and the Navier-Stokes with 1st-order slip boundary condition.

approximately $2.5 \%$ for all Knudsen numbers. This difference causes an oscillation, which is damped out over a few cells. Our study shows that the double-minmod limiter reduces the oscillation to some degree, but the jump at the boundary cell is left unchanged. This jump is not affected by grid refinement either; the refinement only makes the oscillatory domain smaller.

It is important to mention that all our results presented so far are obtained with the $1-C$ boundary condition. Numerical experiments indicate that BGK-sols and 13M- sols with the 2-C model are not significantly different. It turns out that this indifference stems from the nature of linearized Couette flow:

$$
\begin{aligned}
\widetilde{T} & =T^{\mathrm{w}} \\
\sqrt{\frac{R \widetilde{T}}{2 \pi} \frac{\widetilde{q_{y}}}{5 \widetilde{p} R \widetilde{T}}} & \ll 1 \\
P_{y y} & \simeq p
\end{aligned}
$$

From Table 1, with these assumptions, we obtain $C_{1} \simeq 1$ and $C_{2} \simeq 1$ from both solidboundary models. The two models are indeed almost identical. 


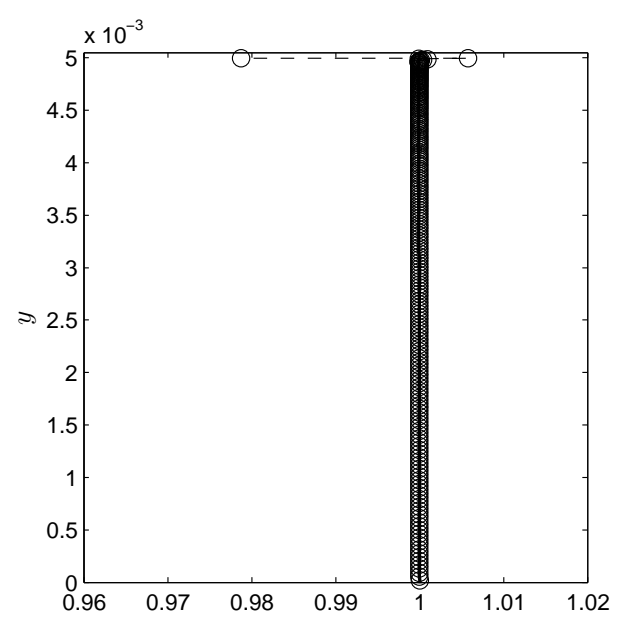

(a) $K n=0.01$

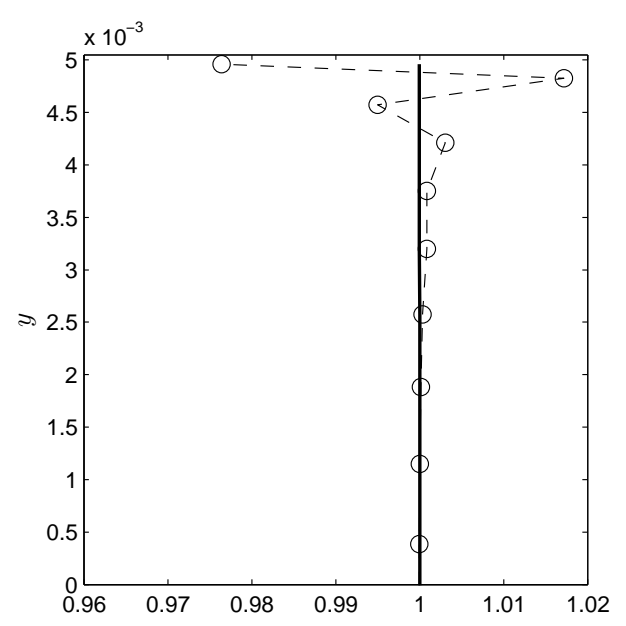

(c) $K n=1$

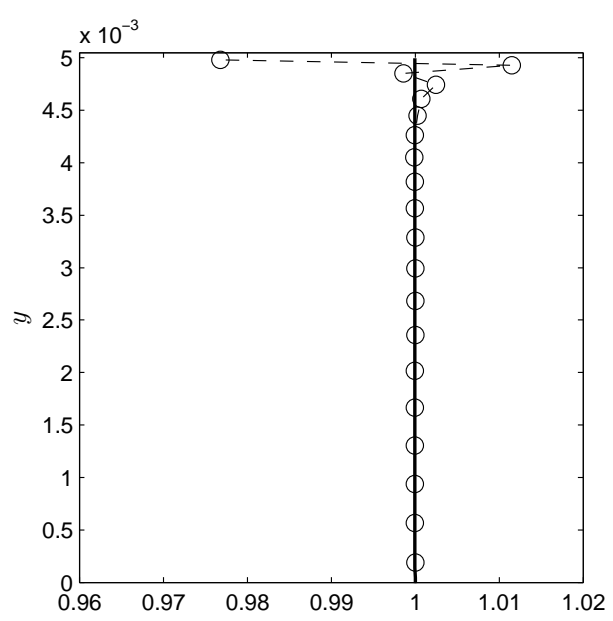

(b) $K n=0.1$

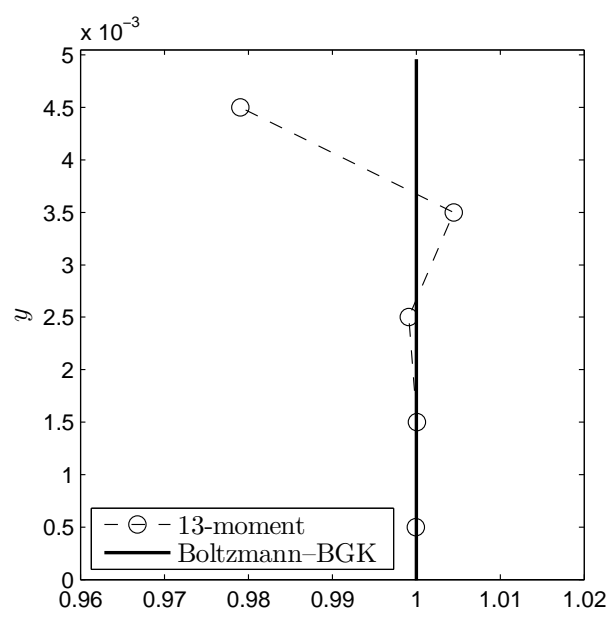

(d) $K n=10$

Figure 3: Profiles of $P_{x y}(y) / P_{x y}(0)$ with $1-C$ boundary condition.

\section{Conclusion}

In this study we present steps towards an accurate solid-boundary condition for Grad-type moment systems. The original approach by Grad produces an inconsistent set of boundary conditions. For any particular flow quantity, we can derive a set of multiple, and different, conditions. Because further constraints are lacking, one can not pick the correct condition out of these. After explaining the root cause, we present two alternative formulations, which are called $1-C$ model and $2-C$ model.

To validate our proposed alternatives, we simulate linearized Couette flow for $K n=0.01$, 
0.1, 1 and 10. The governing system is Grad's 13-moment system and both solid-boundary models are tested. Results are then compared to numerical solutions of the Boltzmann equation, as well as Navier-Stokes solutions obtained with the 1st-order slip condition.

Regarding the ability to predict solutions of the Boltzmann-BGK equation: compared to Navier-Stokes predictions, the moment system has (i) better slip-velocity estimation; (ii) comparable shear stress, $P_{x y}$; and (iii) not as good a prediction for velocity gradients in the middle of the channel.

Because of the nature of linearized Couette flow, solutions from both solid-boundary models are indistinguishable. Our next step will be to apply both boundary models to problems having a higher degree of non-equilibrium, such as Poiseuille flow or Couette flow with a strong heat flux between the two moving walls due to a difference in temperature.

\section{References}

${ }^{1}$ Grad, H., "On the Kinetic Theory of Rarefied Gases," Communications on Pure and Applied Mathematics, Vol. 2, No. 4, 1949, pp. 331-407.

${ }^{2}$ Suzuki, Y. and van Leer, B., "Application of the 10-moment model to MEMS flows," 43rd AIAA Aerospace Sciences Meeting and Exhibit, Reno, Nevada; USA, Jan. 10-13, 2005, AIAA Paper 2005-1398.

${ }^{3}$ Suzuki, Y., Discontinuous Galerkin Methods for Extended Hydrodynamics, Ph.D. thesis, The University of Michigan, 2008.

${ }^{4}$ Khieu, L., Suzuki, Y., and van Leer, B., "An Analysis of a Space-Time Discontinuous-Galerkin Method for Moment Equations and Its Solid-Boundary Treament," 19th AIAA Computational Fluid Dynamics Conference, San Antonio, Texas; USA, June 22-25, 2009, AIAA Paper No. 2009-3874.

${ }^{5}$ Suzuki, Y., Khieu, L., and Leer, B. v., "CFD by first order PDEs," Continuum Mechanics and Thermodynamics, Vol. 21, No. 6, 2009, pp. 445-465.

${ }^{6}$ Chu, C. K., "Kinetic-Theoretic Description of Formation of a Shock Wave," Physics of Fluids, Vol. 8, No. 1, 1965, pp. 12-22.

${ }^{7}$ Chu, C. K., "Kinetic-Theoretic Description of Shock Wave Formation. II." Physics of Fluids, Vol. 8, No. 8, 1965, pp. 1450-1455.

${ }^{8}$ Vincenti, W. G. and Kruger, Jr., C. H., Introduction to Physical Gas Dynamics, Krieger Publishing Company, Malabar, Florida, 1986.

15 of 15 